\title{
KLEBSIELLA PNEUMONIAE (FRIEDLÄNDER'S BACILLUS) INFECTIONS IN INFANCY
}

\author{
BY \\ BELA STEINER and GYULA PUTNOKY \\ From the Department of Paediatrics and the Central Laboratory of Szabolcs-utca Hospital, \\ Budapest, Hungary
}

(RECEIVED FOR PUBLICATION DECEMBER 2, 1955)

Klebsiella pneumoniae infections are uncommon in premature and maternity units as well as in paediatric wards. Hospital epidemics are exceptional, only some five published reports being known to us. Kliewe (1930) reports an epidemic in which pneumonia developed in 11 or 12 infants in a single ward, Klebsiella having been cultured from the pharynx of all 11. Two infants died. Of the remaining 130 children in the institution, only one had a positive throat culture. Jampolis, Howell, Calvin and Leventhal (1932) witnessed an outbreak of diarrhoea in 34 newborn infants caused by Bacillus mucosus. There were 14 deaths. Walcher (1946) found Bacillus mucosus in 12 cases of infantile diarrhoea. Obrinsky, Dormont, Fowler and Ruhstaller (1950) recorded 10 infantile infections caused by the Friedländer aerogenes group of organisms, with five deaths. Očko-Četkovič and Vesenjak-Zmijanac (1953) report on fulminant pneumonia and grave diarrhoeal disorders caused by Aerobacter aerogenes in premature babies and infants.

In our hospital, during the period between December 7, 1953, and July 1, 1954, positive Klebsiella cultures were obtained from the pharyngeal swabs of 52 premature babies, newborn babies and infants, of two children and two adults, as well as from the mucopurulent discharge in two children with otitis, and from the cerebrospinal fluid of a newborn infant, altogether on 94 occasions. The organism was often isolated in pure culture or was found to be predominant. The infections were severe, moderate or even mild. Notwithstanding its presence in throat cultures, it proved very often entirely harmless. It may be, however, that a Klebsiella population of a purely saprophytic nature assumes pathogenic properties.

Such a high incidence of Klebsiella pneumoniae infections seems to be unrecorded as yet in the paediatric literature, and the publication of our material, therefore, may be of some use.

\section{Differentiation of the Escherichine}

In the classification of Bergey (1948) the Escherichiae include Genus I, Escherichia; Genus II, Aerobacter and Genus III, Klebsiella. The absence of a capsule permits us in most cases to differentiate Escherichia coli from A. aerogenes and Klebsiella pneumoniae. Because the latter two organisms show a close morphological, cultural, biochemical and antigenic relationship, their differentiation has been abandoned by numerous authors. Obrinsky et al., for instance, use the term 'FriedlanderAerogenes infections'.

We, however, do not think that the differentiation of these two micro-organisms should be given up. Even their epidemiological behaviour shows some difference: we have been able to isolate $A$. aerogenes from throat cultures only very rarely and never in succession or in a great number of cases simultaneously, but we did, during our epidemic, isolate Klebsiella pneumoniae repeatedly in numerous cases. According to our observations, the Klebsiella cultures on blood and blood-chocolate agar are characterized by white or greyish-white, sticky, mucoid and more or less transparent colonies. $A$. aerogenes produces mucoid, sticky, white or greyish but definitely less transparent colonies. Microscopically, Klebsiella shows a more uniform and monotonous picture than that of $A$. aerogenes. The Klebsiella strains isolated by us have grown well on blood, blood-chocolate and broth-agar, fermented lactose, dextrose, maltose and mannite; they were, however, unable to produce indol. The methyl-red test was negative, the Voges-Proskauer test often positive. There was often an adequate growth in Koser-citrate-medium. But the $\boldsymbol{A}$. aerogenes strains have shown similar behaviour. The best method of distinguishing these two microorganisms has been the inoculation of white mice: very small quantities of Klebsiella pneumoniae killed the mice within 24 hours, whereas the $A$. aerogenes 
either did not kill them or did so with a substantial delay. Weiss, Eisenberg, Alexander and Flippin (1954) established their aetiological diagnosis on similar grounds; in 24 out of 383 patients affected with pneumonia, the aetiological role of Klebsiella pneumoniae was established by these authors. As our strains have proved lethal to white mice within 24 hours, we shall use the term Klebsiella pneumoniae (Friedländer's bacillus) infections in the present paper.

\section{The Route of Klebsiella preumoniae Infection}

The latest data show that Klebsiella pneumoniae infections in children are on the increase. This may be due either to the greater frequency of this organism or to its greater virulence. The first suggestion is based on the fact that the suppression of penicillinsensitive Gram-positive organisms by antibiotic therapy is followed by the overgrowth of Gramnegative bacteria, and among them of Klebsiella pneumoniae. The observations made by Wilhelm and Orkin (1949), on the other hand, lend support to the other view, since the higher incidence of grave urinary tract infections caused by $A$. aerogenes as observed by these authors might be ascribed to an increased virulence. Neither Obrinsky et al. (1950) nor Weiss et al. (1954) were able to determine whether they faced a real or apparent increase in infections.

The epidemic described by Kliewe (1930) and Jampolis et al. (1932) suggests that the high incidence of Klebsiella infections may be unconnected with antibiotic treatment. The infection may spread by carriage even when no antibiotic treatment has been given. We found in our epidemic that in many cases the throat swabs were positive before any antibiotics were given. It was not at all exceptional to find that Klebsiella pneumoniae disappeared spontaneously from the throat cultures before the start of antibiotic therapy. We have had cases where serial throat swabs yielded alternately positive and negative cultures. The question whether the epidemic had anything to do with local circumstances was also pertinent. Valuable evidence could be gathered from our premature unit. Premature babies born outside our hospital are rarely admitted. Therefore, it was very important in view of the above problem that Klebsiella pneumoniae could be recovered from the throat swabs of two premature babies admitted from two widely separated districts of Budapest. This fact appears to indicate that we were actually faced with a town rather than a local increase in the numbers of this organism.
A case (Steiner, 1954) of Klebsiella pneumoniae meningitis in a newborn baby was the first appearance of this organism in our unit. The second infection occurred only two and a half months later. Infection and disease in the newborn might originate directly from the mother or from the immediate environment. Maternal infection may take place during intra-uterine life, during parturition and after birth. As the infant was isolated from the mother immediately after birth, the latter possibility may be excluded. Attempts at isolating Klebsiella pneumoniae from the throat or genital organs of the mother failed. Thus, a maternal source of infection was highly improbable. Klebsiella pneumoniae could not be isolated either from the throats of premature infants sharing the same ward or from those of newborn babies staying at the same time in the maternity unit. Nor could this agent be grown from the air of these wards or from the throat swabs taken from the nurses in contact with the baby. Unfortunately, no investigations in faeces were carried out. Since the newborn baby had been given penicillin for four days before having been taken ill with meningitis, it was suggested that this therapy might have played a part in the development of Klebsiella pneumoniae meningitis.

Our observations seem to suggest that the higher incidence of diseases due to Klebsiella pneumoniae (and to other facultatively pathogenic Gramnegative organisms) has not yet been adequately explained. Since it has been demonstrated that Klebsiella pneumoniae could be recovered in a great number of premature babies who had received no antibiotic treatment and that local causes could not be detected, it must be assumed that the number of Klebsiella pneumoniae organisms is on the increase. If antibiotic therapy is supposed to play an essential part in this increase, we cannot say that the observations gathered during our epidemic have given much support to this suggestion. In our unit, the epidemic caused by Klebsiella pneumoniae was of sudden onset and it came to a sudden end. The first case appeared on December 7, 1953. Until February 16, 1954, we saw no further case. Throat cultures were positive in March on 27 occasions, in April on 25, in May on 27 and in June on 11; in July and September now and then on one occasion (altogether 94 times). Since the same principles of antibiotic therapy were observed in our unit long before the outbreak of the epidemic and have been followed ever since, we do not believe that antibiotic treatment was responsible for the outbreak of the epidemic or that it has fostered it in any way. Thus, our observations, without giving 
a definite answer to the question of how Klebsiella pneumoniae led to this epidemic, do give, however, an important indication: the infections caused by Klebsiella pneumoniae and other uncommon pathogens are not simply the consequence of antibiotic therapy.

\section{The Pathogenic Role of Klebsiella preumoniae}

The pathogenic role of Klebsiella pneumoniae seems to be different in children and in adults. Klebsiella are a group of encapsulated Gramnegative bacilli, present in the normal flora of the mouth and in the respiratory and intestinal tracts in adults (Beeson, 1954). Therefore, in healthy individuals, they are not thought to assume pathogenic properties. On the other hand, Bloomfield (1921) dwells on the fact that the bacillus is rarely found in the upper air passages of healthy individuals. This is confirmed by the investigations of Weiss et al. (1954). According to Julianelle (1930) and Henriksen (1952), 40 out of the 42 strains 'A' of Klebsiella pneumoniae came from human sources. From these 40 serological types, 33 were causative of pneumonia, one of liver abscess, five were saprophytes of the respiratory tract and one was cultured from the urinary tract. From 12 strains of Type B, seven were cultured from animals, the others from human sources (pneumonia, throat, liver abscess, faeces). Type $C$ is very rare, three strains having been isolated from human pneumonia, one from paranasal sinuses and one from the nose. Both the abovementioned authors conclude that Types $A$ and $C$ are highly pathogenic for man, especially Type A, which is generally the cause of Klebsiella pneumonia. According to Weiss et al. (1954) Type A was causative only in three out of 24 cases of Klebsiella pneumoniae infection. As regards its occurrence in children, less than 30 cases of acute Friedländer pneumonia have been reported up to 1950 (Obrinsky et al., 1950). Even during hospital epidemics it was most exceptional to find this organism in the throat of healthy children (Kliewe, 1930). For this reason, positive throat swabs or sputa may induce the paediatrician to ascribe the actual illness to this agent, and his therapeutic measures will also be guided by this belief. We have found it sometimes very difficult to say whether a positive Klebsiella pneumoniae throat culture had anything to do with the actual illness during the time of an epidemic. We had cases in which a pulmonary infection was present and the nasopharyngeal or laryngeal swabs yielded pure or almost pure growths of Klebsiella pneumoniae, which, however, were not causative. In one of our premature infants the clinical diagnosis of interstitial pneumonia could be made in spite of a positive throat culture. The necropsy confirmed this diagnosis without revealing any sign of Klebsiella pneumoniae infection. In another case, clinical diagnosis pointed to aspiration pneumonia (the bacteriological finding in the throat culture revealed Klebsiella pneumoniae) and as soon as a sunflower seed was removed with the aid of the bronchoscope the child recovered completely.

These observations raise the following questions: When is Klebsiella pneumoniae to be regarded as causative and when as a mere saprophyte? Are there carriers of Klebsiella pneumoniae among infants? Which is of greater aetiological significance, if Klebsiella pneumoniae is isolated from nasopharyngeal swabs in pure growth, or if it is only predominant? What is the significance of small numbers of Klebsiella pneumoniae in nasopharyngeal cultures?

It is certainly not easy to find an answer to these questions, but clinical experience may be of great assistance. Thus, Adams (1952) points out that primary pneumonia of bacterial origin is rare in early infancy, whereas primary pneumonia supposed to be of viral aetiology is characteristic of this age, premature infants being particularly susceptible. Our own experience confirms that many cases of Klebsiella pneumonia may be overlooked if only organisms involving destruction or abscess formation are taken into account. The results of experimental investigations do not recommend us to attach too much value to the presence of bacteria in throat cultures. Blake and Cecil (1920) demonstrated in animal experiments that pure cultures of Streptococcus haemolyticus and Diplococcus pneumoniae never cause pneumonia unless introduced by the intratracheal route, whereas influenza bacilli were capable of initiating infection by mere nasal inoculation. According to Robertson (1943), the presence of infected exudate in the upper respiratory tract is harmless to the lungs; only when passing the epiglottic barrier does it lead to pulmonary infection. In the case of droplet infection, the agent must reach the terminal airways in large numbers, without the possibility of being eliminated, in order to develop pneumonia. Bacterial pneumonia is preceded, as a rule, by some disorder of the respiratory passages.

In some cases it is easy to ascertain whether Klebsiella pneumoniae has been causative or merely a saprophyte. For instance, in the case of a premature infant weighing 2,000 g., pure cultures of Klebsiella pneumoniae were obtained from the nasopharynx on the tenth day of its stay in our unit, though the weight curve showed a steep rise and its condition was excellent. Klebsiella pneumoniae 
disappeared from the throat without any treatment. Clearly the organism is not necessarily pathogenic in prematures. This premature baby was a carrier. This observation having been confirmed on other premature and newborn babies, we do not believe that healthy carriers of Klebsiella pneumoniae require any preventive treatment.

In other cases our task was more difficult. For instance, Klebsiella pneumoniae and Gram-positive cocci could be grown from the throat of a newborn baby weighing $2,820 \mathrm{~g}$. Persistent vomiting for over five days was remarkable. We wondered whether Klebsiella pneumoniae could be held responsible for it, but this proved not to be the case, since the vomiting still continued when the nasopharyngeal cultures no longer contained Klebsiella pneumoniae and Gram-positive cocci or Gramnegative bacilli only in small numbers.

A premature baby weighing $1,500 \mathrm{~g}$. was thriving satisfactorily, having gained $500 \mathrm{~g}$. within a month. When the baby was 5 weeks old, a routine examination revealed haemolytic streptococci, Grampositive cocci and diplococci but no Klebsiella pneumoniae in nasopharyngeal cultures. Three days later a temperature of $38^{\circ} \mathrm{C}$., with rapid breathing and harsh respiration had developed. Penicillin induced apyrexia in 24 hours and the development of the infant remained undisturbed. At the date of the onset of temperature Klebsiella pneumoniae could be grown in pure culture from the nasopharyngeal swab. Though this bacterium is insensitive to penicillin, it could no longer be demonstrated after treatment. Since the patient recovered as a result of penicillin treatment, and Klebsiella pneumoniae, which is known to be unresponsive to penicillin, disappeared from the throat during treatment, we do not believe that in this case Klebsiella pneumoniae played any part as an infecting agent.

A 1-day-old premature baby was Klebsiella positive without presenting any symptoms when admitted to our unit. A few days later pneumonia developed and the patient was started on penicillin. Twenty-four hours later the throat culture yielded, apart from a few Gram-positive cocci, Klebsiella pneumoniae. On the basis of this finding, streptomycin was substituted for penicillin. A 10 days' course of treatment resulted in complete cure. However, three weeks later the throat cultures were again Klebsiella-positive. Now dyspepsia developed but it responded to streptomycin and threomycin. Thus, in this case positive Klebsiella pneumoniae cultures were accompanied by pneumonia, dyspepsia and perfect condition and development. Whereas the aetiological role of Klebsiella pneumoniae is probable, it does not conclusively cause dyspepsia.
The patient might have been only a carrier of bacilli at the time when his development was satisfactory.

Our experience favours the view that Klebsiella pneumoniae in the throat even of the youngest baby has not necessarily pathological consequences. In each individual case it must be decided whether we are faced with the causative agent or whether we have to deal with carriers only. In our ample material we have met numerous carriers. The question whether there is any connexion between Klebsiella pneumoniae demonstrated in the nasopharynx and the actual illness must be raised in each individual case, in particular during epidemics. Klebsiella pneumoniae obtained in pure growth may have a greater aetiological significance than in cases where it is but a member of the nasopharyngeal flora. But here we must state clearly that, in our opinion, Klebsiella pneumoniae in the nasopharynx, whether in pure culture or only as the predominant organism, is by no means indicative of any connexion existing between the actual illness and this agent. Thus, statements to be found in textbooks (Nelson, 1954), according to which a bacteriological finding is diagnostic of Klebsiella pneumonia, need revision.

In case of mixed bacterial cultures, it would have been necessary to separate them from one another and to carry out sensitivity tests separately with each. However, even with this procedure we would not have been able to decide in each individual case which of the various strains were responsible for the symptoms observed. Whereas formerly the simultaneous aetiological role of several agents in bronchopneumonia was generally acknowledged, to-day it is, on the contrary, believed that even if several organisms are found it is only one of them which causes the disease (Adams, 1952). In cases where a mixed culture is found, the role of Klebsiella pneumoniae would be ascertained, apart from the clinical course, by the presence of Types 1-6 of groups $\mathrm{A}$ or $\mathrm{C}$ but mostly by the demonstration of specific antibodies in the patient's serum.

The observations according to which the epidemic described by Kliewe (1930) was confined to pneumonia cases and that witnessed by Jampolis et al. (1932) to diarrhoeal cases, directs our attention to the problem of typing Klebsiella pneumoniae. Unfortunately, it is rather difficult to separate the different types of Klebsiella pneumoniae, of which more than 50 are known to-day, not only because there are no specific antisera available, but also because capsulated bacilli are not easy to agglutinate. According to Grotts (1949) there are types of Friedländer bacilli which are agglutinable and others which are not. The serological difficulties are 
clearly shuwn by the investigations of Henriksen (1952). Only 141 out of 189 capsulated strains responded to the antisera of generally known types, but the typing was possible in 90 strains $(47 \cdot 6 \%)$ only; in $46.1 \%$ this was not feasible with 61 typesera.

In our opinion, the actual state of knowledge does not allow us to consider the causal role of Klebsiella pneumoniae in children as proven unless (1) this organism is cultured from blood or from pleural effusion, (2) demonstrated in a cerebrospinal fluid which shows inflammatory symptoms, (3) grown from urine taken under sterile precautions and the number of white blood cells in the urine is increased, (4) demonstrated in the faeces during a dysentery epidemic among children, and the carrier or carriers of Klebsiella pneumoniae having been removed, the epidemic comes to an end, and (5) the significance of a positive nasopharyngeal culture of Klebsiella pneumoniae in the diagnosis of Klebsiella pneumonia is different when we are faced with an isolated case or with a mass infection, This problem will be discussed in another report (Steiner, in the press).

\section{Summary}

An outbreak of infection Klebsiella pneumoniae in infants is described. Antibiotic therapy did not seem to play any part. In the course of an epidemic the fact that Klebsiella pneumoniae is only a facultative organism must be taken into account. It should be considered in each case whether the presence of Klebsiella pneumoniae in the nasopharynx is in any way connected with the actual disease. In the differential diagnosis of sepsis, meningitis, diseases of the urinary tract and pneumonia the aetiological role of Klebsiella pneumoniae has to be considered as being more frequent than before.

\section{REFERENCES}

Adams, J. M. (1952). Brennemann's Practice of Pediatrics, Vol. 2, Ch. 48, pp. 1-37. Hagerstown, Maryland.

Beeson, P. B. (1954). In Principles of Internal Medicine, ed. Harrison, T. R., 2nd ed., p. 847. London.

Bergey, D. H. (1948). Manual of Determinative Bacteriology, 6th ed. Ed. Breed, R. S., Murray, E. G. and Hitchens, A. P. Baltimore.

Blake, F. G. and Cecil, R. L. (1920). J. exp. Med., 31, 403.

Bloomfield. A. L. (1921). Bull. Johns Hopk. Hosp., 32, 33.

Grotts, B. F. (1949). J. Pediat., 34, 174.

Henriksen, S. D. (1952). Acta path. microbiol. scand., 30, 230.

Jampolis, M., Howell, K. M., Calvin, J. K. and Leventhal, M. L (1932). Amer. J. Dis. Child., 43, 70.

Julianelle, L. A. (1930). J. exp. Med., 52, 539.

Kliewe, H. (1930). Zbl. Bakt., 1, Abt. Órig. 116, 92.

Nelson, W. E. (1954). Textbook of Pediatrics, 6th ed., p. 822 Philadelphia and London.

Obrinsky, W., Dormont, R. E. Fowler, R. E. L. and Ruhstaller, F. (1950). Amer. J. Dis. Child., 80, 621.

Očko-Četkovič, S. and Vesenjak-Zmijanac, J. (1953). Ann. paediat. (Basel), 181, 17.

Robertson, O. H. (1943) Ann intern. Med, 18, 1.

Steiner, B. (1954). (a) Orv. Hetil., 95, 1100; (b) Ther. hungar., 2, 23.

. Acta Medica (Budapest). In the press.

Walcher, D. N. (1946). J. clin. Invest., 25, 103.

Weiss W.. Eisenberg, G. M., Alexander, J. D. and Flippin, H. F (1954). Amer. J. med. Sci. 228, 148.

Wilhelm, S. F. and Orkin, L. A. (1949). J. Urol., 61, 131. 